\title{
Cristo si è fermato a Eboli \\ Gagliano e il parco letterario di Aliano: metamorfosi di una memoria
}

\section{Francesca R. Uccella}

Universitat de Girona

\begin{abstract}
La relazione fra Carlo Levi, la sua opera Cristo si é fermato a Eboli ed il paese di Aliano ha subito dei cambiamenti dal momento della pubblicazione del libro (1945) fino all'istituzione del Parco Letterario Carlo Levi (2001). L'esperienza e il ricordo del confino influenzarono molto la formazione dell'intellettuale antifascista e marcarono profondamente le sue successive scelte letterarie e politiche. Di pari intensità fu la reazione che il libro suscitò nel piccolo centro lucano. Passati ormai più di cinquanta anni, l'iniziale posizione critica e distante degli alianesi si é via via modificata fino a permettere, grazie al patrimonio letterario lasciato da Levi, una valorizzione culturale del territorio.
\end{abstract}

Parole chiave: Carlo Levi, Cristo si é fermato a Eboli, Parchi Letterari, Aliano, confino, patrimonio letterario.

\section{Abstract}

The relationship between Carlo Levi, his work Christ Stopped at Eboli and the village of Aliano, has changed a great deal between the first publication of the book in 1945 and the establishment of the Parco Letterario Carlo Levi in 2001. The experience and the memory of Levi's exile in Aliano profoundly affected his intellectual development and deeply influenced both his subsequent literary and political choices. When the book first appeared, it provoked an intense reaction, but fifty years later the initial response from the critics had changed to the extent that an easier and more constructive relationship between people from Aliano, the author and his masterpiece was possible.

This process culminated in a cultural endowment called the Parco Letterario Carlo Levi, which aims to improve links between the region territory and its literary heritage.

Key words: Carlo Levi, Christ Stopped at Eboli, Parchi Letterari, Aliano, exile, literary heritage.

L'ambito generale di questo scritto è costituito dall'analisi della relazione che si stabilisce fra opera letteraria e territorio, tema che trova la sua origine nell'esigenza di esplorare uno degli aspetti della cosiddetta geografia letteraria, 
disciplina basata sul vincolo che ogni autore mantiene con i propri luoghi d'ispirazione. In questa sede si riferirà del rapporto fra il libro Cristo si è fermato a Eboli di Carlo Levi ed Aliano, paese lucano in cui l'autore fu confinato e del quale raccontò nelle sue pagine; in particolare della significativa evoluzione del rapporto fra il testo, pubblicato da Einaudi nel 1945, e il paese teatro delle vicende narrate.

Carlo Levi fu destinato al confino ad Aliano dal regime di Mussolini a causa delle sue idee ed attività politiche. Lo scrittore torinese non visse in paese più di otto mesi, fra il 1935 e il 1936, ma la sua esperienza fu così forte da lasciare segni profondi non solo in lui, ma anche nella memoria personale e collettiva degli abitanti del piccolo centro. A dieci anni dal periodo di confino, Levi si decise a scrivere Cristo si è fermato a Eboli, un testo che, dal momento della pubblicazione ebbe un grande successo e servì a mostrare ad un gran numero di lettori le precarie condizioni di vita dei contadini meridionali negli anni Trenta.

Da sei anni ormai nel paese lucano è stato istituito un Parco Letterario, ovvero un ente che si occupa di gestire la memoria di Carlo Levi, ancora molto forte, e legata ai luoghi che egli descrisse e con i quali stabilì una relazione speciale, come la casa di confino, le cosiddette case con gli occhi o il piccolo cimitero nel quale è sepolto.

In queste pagine cercherò di evidenziare i cambiamenti avvenuti nel rapporto fra Cristo si è fermato a Eboli, il suo autore ed Aliano, da un lato tenendo conto della vasta bibliografia di taglio strettamente antropologico, dall'altro ricorrendo a un buon numero di interviste svolte durante un prolungato lavoro sul campo. ${ }^{1}$

I preliminari della ricerca rientrano nell'ambito dell'antropologia letteraria, cioè in quel settore della disciplina antropologica contraddistinto dall'attenzione riposta sull'uso di differenti tipi di testi — romanzi, racconti, saggi, diari personali, epistolari e memorie- per analizzare fenomeni culturali e sociali.

Il rapporto di convivenza fra Carlo Levi e gli abitanti di Aliano iniziò all'arrivo dello scrittore nel 1935, ad estate inoltrata, e si interruppe in modo improvviso, quanto inaspettato, il 26 maggio 1936, giorno in cui Carlo Levi lasciò il piccolo paese fra i calanchi, una settimana dopo la liberazione dei confinati politici disposta dal Ministro degli Interni in occasione della proclamazione dell'Impero.

Il Cristo è nato in due momenti successivi, a distanza di dieci anni l'uno dall'altro. Il primo risale sicuramente al soggiorno dell'autore ad Aliano, periodo nel quale Levi visse e osservò la realtà che lo circondava, prendendo tanti

1. Lanalisi del rapporto fra Cristo si è fermato ad Eboli, Carlo Levi ed Aliano ha costituito il tema centrale della mia tesi di laurea, intitolata "Come una pietra in uno stagno". Le strategie della memoria del "Parco Letterario Carlo Levi» di Aliano, discussa presso l'Università degli Studi di Roma "La Sapienza» nel marzo 2003. Il lavoro sul campo cui si fa qui riferimento si è svolto ad Aliano fra i mesi di febbraio e luglio del 2001. 
appunti, poi confluiti nella stesura delle famose pagine. Non si tratta solo di annotazioni scritte, ma anche dei numerosi quadri che egli dipinse e delle poesie che compose durante la sua permanenza ad Aliano. ${ }^{2}$ Questi modi di fissare immagini e sensazione lo aiutarono a ricostruire il suo universo lucano, quando durante l'inverno del tragico 1943 si trovò nuovamente costretto in una stanza, in una Firenze assediata dai tedeschi. La permanenza in questa città, in un appartamento di Piazza Pitti, costituì il secondo momento dell'elaborazione del testo, quello della vera e propria scrittura, che si configurava allora come unico autentico antidoto alla morte. ${ }^{3}$

Nel libro convergono emozioni, sensazioni di dieci anni di storia, personale e collettiva. La guerra che infuria spinge Carlo Levi a «riandare con la memoria a quell'altro mondo», ${ }^{4}$ la Basilicata ed Aliano, che per lui erano stati autentiche scoperte di un universo "opposto a quello dell'alienazione della modernità industriale». 5

Come apprendiamo dalla lettera scritta a Giulio Einaudi — suo editore da sempre- come prefazione all'edizione del Cristo del 1963, Levi intese l'universo di Aliano come una rivelazione della realtà, di «ignoti linguaggi, lavori, fatiche, dolori, miserie e costumi, non soltanto animali e magia, e problemi antichi non risolti, e una potenza contro il potere, ma l'alterità presente, l'infinita contemporaneità, l'esistenza come coesistenza, l'individuo come luogo di tutti i rapporti, e un mondo immobile di chiuse possibilità infinite, la nera adolescenza dei secoli pronti ad uscire e muoversi, farfalle dal bozzolo». ${ }^{6}$

Queste «farfalle» che si liberano sono anche portatrici di un messaggio pratico, rivolto soprattutto alla borghesia progressista, un messaggio che vuole spingere gli intellettuali a rendersi conto dell'esistenza di un Sud ignoto, luogo di partenza potenziale di una nuova rivoluzione contadina, per merito «degli uomini nuovi, dei piccoli, degli oscuri» che Levi ebbe modo di conoscere e grazie ai quali avrebbe potuto formarsi ampliando, inaspettatamente, il suo "campo visivo e d'azione».

Per Levi la Lucania diventò il simbolo di uno stato di oppressione, emarginazione ed esclusione subita anche da altri in altre zone della terra: «dalla Lucania di Grassano e Gagliano alle lucanie del mondo».7 Questa espressione, coniata da Levi in una particolare occasione, la guerra nel Vietnam (19591973), diventò ad Aliano una formula quasi proverbiale: «Aliano diventa il

2. Pia Vivarelli (a cura di), Carlo Levi. Paesaggi 1926-1974. Lirismo e metamorfosi della natura, Corigliano Calabro Scalo: Meridiana Libri, 2001.

Carlo Levi, Poesie Inedite 1934-1946, Foggia: Mancosu, 1990.

3. Levi non scrisse tutto il Cristo nella stanza segreta di piazza Pitti, ma la stesura del libro seguì i suoi frequenti spostamenti fra Firenze e Fiesole, dove fu ospite, in aprile, della famiglia Nerini.

4. Carlo Levi, Cristo si è fermato a Eboli, Torino: Einaudi, 1990 [1945], p. 3.

5. Gigliola De Donato, Sergio D’Amaro, Un torinese del sud: Carlo Levi. Una biografia, Milano: Baldini \& Castoldi, 2001, p. 163.

6. Carlo LeVI, Cristo, cit., p. XVII.

7. Carlo LeVI, Presentazione a Cristo si è fermato a Eboli, Torino: Einaudi, 1968, p. IX. 
simbolo, di tutte le Aliano sparse nel mondo». La frase significa per gli alianesi di oggi sapere che Levi innalzò il loro paese a simbolo di tutti quei luoghi dove le condizioni ambientali e sociali rendono la vita tanto difficile. Il contadino lucano diventa così per Levi uno dei tanti protagonisti esemplari delle molte lucanie della terra, sia in altre regioni d'Italia, sia in diversi luoghi nel mondo. ${ }^{8}$

La frase è stata completamente isolata dal suo contesto storico ed ora è ripetuta infinite volte, dalle guide turistiche, dal parroco del paese - attivo promotore culturale - e da chiunque altro si trovi a spiegare il senso del libro. Con la spiegazione data ai visitatori, gli alianesi sembrano voler riscattarsi dallo stato in cui Levi li aveva descritti nel testo, allontanandosi da quella connotazione negativa attribuita al paese e alla sua comunità. L'acquisizione di questo carattere universale aiuta Aliano a confondersi con mille altri piccoli centri di chissà quali continenti.

Vediamo ora come le cose cambiarono radicalmente al momento della pubblicazione di Cristo si è fermato a Eboli (1945). Quando il libro uscì, riscosse un grande successo. Uno dei primi a farne un'ottima critica fu Eugenio Montale che sul Mondo del febbraio 1946 collocò il Cristo entro «ampie coordinate letterarie» sottolineandone «'equilibrio tra tentazione decadentistica e sapienza artistica». ${ }^{9}$ A quello di Montale fecero seguito altri articoli di Vittore Branca, Enrico Falqui, Rosario Assunto e Giovanni Russo. ${ }^{10}$

Più guardinga è invece la critica di coloro che Carlo Levi stesso inizierà di lì a poco a chiamare $\mathrm{i}$ «luigini comunisti»: ${ }^{11}$ Giuseppe Petronio, Carlo Muscetta, Mario Alicata, Carlo Salinari. Muscetta, ad esempio, lo accusa di aver descritto Aliano come egli lo aveva voluto immaginare e sognare, esaltandone gli aspetti più funzionali alla sua visione del Sud. ${ }^{12}$ Nonostante le critiche, il Cri-

8. Cfr. Carlo LeVI, Le parole sono pietre. Tre giornate in Sicilia, Torino: Einaudi, 1955; Id., Il futuro ha un cuore antico. Viaggio nell'Unione Sovietica, Torino: Einaudi, 1956; ID., Tutto il miele è finito, Torino: Einaudi, 1964.

9. Gigliola De Donato, Sergio D’Amaro, Un torinese del sud: Carlo Levi. Una biografia, Milano: Baldini \& Castoldi, 2001, p. 175.

10. Cfr. rispettivamente: La Nazione del Popolo (21 febbraio 1946); La Lettura (28 febbraio 1946); Italia Libera (18 agosto 1946); La Voce (11 settembre 1946).

11. Carlo LevI, L'Orologio, Torino: Einaudi, 1950, p. 166. «E i Luigini, chi sono? Sono gli altri. La grande maggioranza della sterminata, informe, ameboide piccola borghesia, con tutte le sue specie, sottospecie e varianti, con tutte le sue miserie, i suoi complessi d'inferiorità, i suoi moralismi e immoralismi, e ambizioni sbagliate, e idolatriche paure. Sono quelli che dipendono e comandano; e amano e odiano le gerarchie, e servono e imperano...».

12. Cfr. Carlo MusCETTA, "Leggenda e verità di Carlo Levi», La fiera letteraria, 14 novembre 1946: «Nell'era tumultuosa del totalitarismo, l'artista che segue una sua razionale utopia libertaria non può non essere attratto dalla Città delle Tenebre», in Gigliola DE DONATO, Sergio D’Amaro, Un torinese del sud: Carlo Levi. Una biografia, Milano: Baldini \& Castoldi, 2001, p. 175.

Angelo Colangelo, intervista del 30 maggio 2001, in Francesca R. UCCELLA, "Come una pietra in uno stagno". Le strategie della memoria del "Parco Letterario Carlo Levi» di Aliano. Tesi di Laurea in Etnologia delle Culture Mediterranee, Università degli Studi «La Sapienza» di Roma, marzo 2003, p. 86. In disaccordo con la posizione di Muscetta, Colan- 
sto piacque al pubblico e, fra il febbraio e l'agosto 1945, ne uscirono tre edizioni. Ben presto arrivarono le prime traduzioni e il testo diventò in breve tempo uno dei libri più venduti negli Stati Uniti. ${ }^{13}$

Il libro produsse grande effetto sui lettori e stimolò critiche e dibattiti. Grazie al suo evidente carattere di denuncia sociale, esso fu inserito nel filone della tradizione meridionalistica per la penetrazione antropologica del mondo descritto. ${ }^{14}$ Venivano infatti individuati gli effetti negativi di quell'immobilismo che sembrava fissare la civiltà contadina in un tempo arcaico, giudicato anche come possibile risultante d'una visione eccessivamente mitica e sentimentalistica. L'attenzione preminente del personaggio-narratore, concentrato a creare la leggenda di se stesso, non intaccò la relazione profonda che Levi instaurò con il mondo contadino e con l'esperienza umana, sociale, politica vissuta. ${ }^{15}$

Anche chi, come Asor Rosa, individuava nella Basilicata reale l'oggetto principale del libro, non mancava tuttavia di sottolineare la soggettività della rappresentazione leviana del mondo contadino. ${ }^{16}$ Essa non comportava comunque, per il critico, il tradimento dell'impianto storico-sociologico del discorso «a favore della bella pagina descrittiva, del colore locale, del puro e semplice folklore». Il Cristo si fonda, infatti, su questo spiccato interesse e, nonostante la sua propensione all'irrazionale e al mitico, Levi rielabora i temi da lui affrontati con una "forza rappresentativa e una serietà culturale» assente nella maggior parte degli scrittori progressisti a lui contemporanei. ${ }^{17}$

La forte tendenza estetizzante, che si manifesta nella ricerca dell'immagine, nel richiamo all'arcaico, alla civiltà greca e alle antiche pratiche magiche, è controbilanciata da un naturale slancio ad aprirsi ai suoi simili, ciò che permette a Levi di stabilire un proficuo contatto di scambio con gli alianesi, anche se, talvolta, con qualche condiscendenza. La civiltà contadina arcaica attirerà

gelo afferma: «Io aggiungerei una cosa sola, che, per molto tempo, anche da parte della critica ufficiale della sinistra, era stata interpretata poco correttamente la posizione di Levi, ma soltanto per pregiudizi di carattere ideologico, perché se si fosse prestata attenzione alla posizione che Levi assunse, sin dal primo momento, si sarebbe avuta la dimostrazione che Carlo Levi poteva essere criticabile per quanto riguardava le soluzioni che egli proponeva riguardo al problema meridionale, ma non poteva certo essere messa in discussione, né l'analisi che egli aveva compiuto, né tanto meno la correttezza e l'onestà intellettuale con cui egli aveva indagato il problema. Sulle soluzioni, naturalmente, poi si può anche discutere, si può anche, alla fine, concludere che quelle di Levi sono delle posizioni utopistiche, quindi irrealizzabili ma sulla sua onestà intellettuale e sulla validità di certe analisi che Levi ha organizzato sulla società meridionale, già nel Cristo e nelle opere successive al Cristo, credo che non si possa assolutamente discutere».

13. Le prime traduzioni furono quella inglese (1946), quella americana (1947) e quella francese (1948) cui ne seguirono altre fra cui quelle al cinese, al giapponese, a russo e al finlandese. Gigliola De Donato, Sergio D’Amaro, Un torinese del sud: Carlo Levi. Una biografia, Milano: Baldini \& Castoldi, 2001, p. 178-179.

14. Giovanni Battista BronZINI, Cultura contadina e idea meridionalistica, Bari: Dedalo, 1982.

15. Ibid., p. 209.

16. Alberto ASOR ROSA, Scrittori e popolo. Il populismo nella letteratura italiana contemporanea, Torino: Einaudi, 1969.

17. Alberto Asor Rosa, op. cit., p. 154. 
Levi e si riverserà nelle sue pagine soprattutto attraverso gli aspetti magici e irrazionali. La magia si presenta come una forma di comprensione e di avvicinamento, chiave indispensabile per arrivare a penetrare il cuore di una realtà nuova e misteriosa. A fianco della magia c'è il mito, c'è il tentativo continuo di renderlo elemento di comunicazione e di interpretazione di tutto ciò che non ricade nell'ambito della logica e della storia. ${ }^{18}$

Levi crea intorno al Sud tutta una serie di questi mithoi, attraverso i quali prendono vita paesaggi, figure, situazioni familiari e sociali. Il suo singolare diario non datato, più che un resoconto, risulta essere una narrazione mitica, in cui le dimensioni del reale e dell'irreale si confondono, anche per effetto di un ritmo teatrale che alterna monologhi, dialoghi e pause di riflessione. ${ }^{19}$

Cardine del pensiero leviano è comunque l'individuazione della civiltà contadina quale complesso globalmente positivo di valori che devono essere riconosciuti e conservati. Proprio in virtù di questa convinzione, egli accetta il suo nuovo paese come un vero, grande modello di umanità che, nel quadro di un ordine nuovo, potrà occupare il suo posto accanto ad altre forme di cultura, senza esserne oppressa e sfruttata.

Ma è la questione meridionale nel suo insieme ad essere oggetto della riflessione di Levi, che parte appunto dal mondo contadino, legato alla terra e ai sentimenti genuini, contrapposto a quello della piccola, miope borghesia di provincia - o dei «Luigini» - colpevole della generale arretratezza socioeconomica tanto quanto i burocrati statali o i grandi proprietari fondiari.

$\mathrm{Vi}$ è anche - e non meno importante- un'altra forma di contrapposizione nella quale lo scrittore si trova immerso: quella tra il suo io, il suo mondo personale e quello di Aliano, che diventano intercomunicanti grazie al carattere di scoperta che Levi imprime alla sua esperienza di confino. ${ }^{20}$ Tale scoperta lo

18. Carlo LeVI, Cristo, cit., p. 98. Esemplare a questo proposito la vicenda della «donna-vacca», contadina che avevava avuto, oltre ad una madre umana, anche una madre vacca ed era una dei misteriosi personaggi che possedevano una natura contemporaneamente umana ed animalesca. Poche righe più avanti Levi parla anche degli uomini lupo: «I sonnambuli diventano lupi, licantropi, dove non si distingue più l'umano dalla belva».

19. Carlo LeVI, Cristo, cit., p. 59. "Ci svegliava una strana voce senza sesso, né timbro, né età, che pronunciava parole incomprensibili. Un vecchio si sporgeva dal bordo della tomba, e mi parlava attraverso le sue gengive sdentate. Lo vedevo contro il cielo, alto e un po' curvo, con delle lunghissime braccia magre, come le ali di un mulino. Aveva quasi novant'anni, ma il suo viso era fuori dal tempo, rugoso e sformato come una mela vizza: fra le pieghe della carne riscecchita brillavano due occhi chiarissimi, azzurri e magnetici. [...] Parlava un dialetto che non era quello di Gagliano, un miscuglio di linguaggi, perché aveva girato molti paesi, ma vi prevaleva la parlata di Pisticci, dove era nato in tempi remotissimi. [...] Ma non ho mai capito se egli veramente mi ascoltasse, o se seguisse soltanto il misterioso gomitolo dei suoi pensieri, che sembravano uscire dalla indeterminata antichità di un mondo animalesco".

20. Carlo LeVI, Cristo, cit., p. 3. «Ma, chiuso in una stanza e in un modo chiuso, mi è grato riandare con la memoria a quell'altro mondo, serrato nel dolore e negli usi, negato alla Storia e allo Stato, eternamente paziente; a quella mia terra senza conforto e dolcezza, dove il contadino vive nella miseria e nella lontananza, la sua immobile civiltà, su un suolo arido, nella presenza della morte». 
avrebbe portato a dare un nuovo indirizzo alla sua vita e alla sua riflessione politico-letteraria, in maniera tanto definitiva da fargli assumere Aliano, e la Basilicata tutta, come sua terra di elezione.

Il carattere particolare che lo scrittore torinese volle imprimere alla sua esperienza, la trasformò in un vero e proprio progetto di conoscenza, stimolato dalla scoperta che gli si palesò improvvisamente davanti agli occhi. Grazie a questo progetto Levi evitò di essere assorbito dal «guscio di noce» e dal «mondo chiuso» di Aliano, attraverso una scelta consapevole e deliberata. La sua vicenda personale e politica lo portò a vivere la condizione canonica del lavoro sul campo propria dell'antropologo, ricercatore solitario che non conosce nulla della realtà in cui si trova immerso, e che inizia un processo di appropriazione graduale di tale realtà. Dopo un certo periodo, lo studioso deve affrontare la scrittura di una monografia. Levi conferisce invece al suo testo un aspetto letterario ricco di esperienze filtrate attraverso i sentimenti. Il risultato è una «monografia» non comparabile a quelle prodotte dagli antropologi del suo tempo, ${ }^{21}$ pur essendo presenti in essa dati sull'articolazione dei grandi momenti della vita collettiva: produzione delle risorse materiali, parentela e famiglia, socialità, potere politico, riti collettivi e sistema di valori.

Lo stupore, l'emozione e la curiosità quasi morbosa che può cogliere chi sente di stare in procinto di scoprire qualcosa, viene incanalata e ridimensionata in momenti di scrittura successivi. ${ }^{22}$

Subito dopo la pubblicazione del Cristo Levi si adoperò affinché la sua distribuzione editoriale al Sud fosse efficace. Ad Aliano il libro suscitò una reazione quasi immediata e non positiva la cui eco, seppur modificata, è arrivata fino ai nostri giorni.

Secondo quanto affermano diversi intervistati, il motivo principale della cattiva accoglienza del libro, era costituito dal fatto che gli alianesi si sentivano offesi dalla maniera in cui Levi aveva rese pubbliche le loro misere condizioni di vita.

Un esempio chiaro di ciò che successe dopo l'arrivo del libro in paese è rappresentato dall'intervista del nipote del vecchio podestà Magalone. Secondo il suo parere, Don Luigino Magalone avrebbe spinto i compaesani a credere che Levi avesse scritto il falso sul loro conto proprio perché non aveva gradito il ritratto che lo scrittore ne aveva fatto nel Cristo.

A questo proposito, è fondamentale considerare che, all'epoca del confino di Carlo Levi, la stragrande maggioranza della popolazione alianese era analfabeta, eccezion fatta per i medici, il podestà, il farmacista, l'impiegato della posta e pochi altri. La maggior parte degli abitanti era quindi impossibilitata a leggere il Cristo; i pochi che erano in grado di farlo, ne diffondevano versioni del tutto parziali.

21. Per tutti confronta: Giuseppe Cocchiara, Genesi di leggende, Palermo: G. B. Palumbo, 1949 o Ernesto De MarTinO, Sud e magia, Milano: Feltrinelli, 1959.

22. Riguardo alla scrittura antropologica, cfr. James Clifford, George MARCus (a cura di), Scrivere le culture. Poetiche e politiche in etnografia, Roma: Meltemi, 1997. 
In effetti, proprio questi erano il bersaglio della critica più forte di Levi e pertanto avevano tutto l'interesse a diffondere l'opinione che il libro fosse offensivo e denigratorio nei confronti di tutti, sia dell'élite sia anche dei contadini.

L'attenzione si concentra soprattutto su due dei protagonisti delle vicende alianesi degli anni '30: uno dei due medici del paese, che l'autore nel Cristo chiama Gibilisco, e quel Don Luigino che doveva ispirare Carlo Levi la famosissima divisione fra Contadini e Luigini. ${ }^{23}$ Secondo Colangelo, il podestà Magalone era stato descritto «in chiave negativa, riguardo all'aspetto umano e professionale, perché meglio fosse rimarcata la sua negatività sul piano politico», facendone risaltare «l'ipocrisia e la perfidia». ${ }^{24}$ Tuttavia è interessante notare che il Cristo non fu ben accetto neanche presso alcune di quelle persone descritte con maggiore affetto, esaltandone le virtù, senza risparmiare i tratti forti, positivi e negativi, sia fisici che morali.

Esempio di tale cattiva accettazione fu la reazione avuta da Giulia la Santarcangelese dopo aver saputo del contenuto delle pagine dedicatele da Levi. Fin dal principio lo scrittore fa di lei una descrizione ricca di elementi che dimostrano il suo interesse particolare per quella donna forte e misteriosa. Raccontò diffusamente anche della sua vita privata, di come aveva condotto la sua esistenza prima dell'arrivo in paese. Credo che più che la sua caratterizzazione, fosse il racconto delle sue vicende personali a mal disporre la donna nei confronti di colui che, per un buon numero di mesi, era stato il suo datore di lavoro.

Ciò trova conferma in un articolo, uscito su La Gazzetta del Mezzogior$n o,{ }^{25}$ in cui si legge una breve intervista ad uno dei figli della Santarcangele-

23. Cfr. Carlo LeVI, L'Orologio, cit., p. 165. «Ecco: i due veri partiti che, come direbbero nel Mezzogiorno, si lottano, le due civiltà che stanno di fronte, le due Italie, sono quella dei Contadini e quella dei Luigini. Contadini e Luigini, [...] ecco i due movimenti, nemici e impenetrabili; ecco le due sole Categorie della nostra storia. Contadini e Luigini, Luigini e Contadini!» E più avanti ( ibid.) «Quanto ai Luigini [...] li chiamo così dal nome di un personaggio fantastico, che, non per colpa o merito suo, li rappresenta completamente, in un libro che tu conosci di certo: quel Don Luigino, podestà e maestro di scuola di un villaggio meridionale che tu sai.»

24. Angelo Colangelo, Gente di Gagliano, ritratti di personaggi leviani, Matera: Circolo Culturale Nicola Panevino, 1994, p. 20.

25. «Quella povera donna con lo scialle nero», Gazzetta del Mezzoggiorno, 9 dicembre del 1990. «Mia madre era analfabeta ..., non sapeva leggere. Quando è uscito il libro qualcuno glielo ha letto. Ė rimasta molto dispiaciuta. Non avrebbe voluto il suo nome, la sua storia, le sue miserie... mia madre era una povera donna... era povera...senza marito». E anche in A. ColANGELO, Gente di Gagliano cit., p. 34, «Sempre il figlio Luigi, poi, senza neppure nominarlo, ha manifestato di serbare per lui un forte risentimento nei confronti di Carlo Levi, che sarebbe stato detestato dalla stessa Giulia per la gratuità e l'infondatezza di molte affermazioni sul conto della vita sentimentale di quest'ultima dopo il matrimonio con un giovane leccese, Nicola Venneri, che la sposò nel 1910». Non solamente i figli parlano dell'atteggiamento risentito di Giulia Venneri. È lo stesso scrittore a narrarci della difficoltà del loro primo incontro dopo la pubblicazione del Cristo. Cfr.: "Giulia, la strega contadina, e la sola che, tra i saluti, i sorrisi, e le lacrime di tutti, non mi vuole vedere, si è chiusa in casa: teme di essere fotografata. Poi, dopo lunghe trattative del medico condotto che mi accompagna, e un debito compenso, mi apre la porta in cima alla scala di pietra, e perfino mi abbraccia», Carlo LEVI, Un volto che ci somiglia. L'Italia com'era, Roma: edizioni e/o, 2000, p. 120. 
se, il quale racconta della reazione dispiaciuta della madre al momento dell'uscita del libro, quando Giulia venne a sapere cosa era stato scritto di lei e della sua vita privata.

Analoghi i sentimenti di Donna Caterina, ${ }^{26}$ sorella di Don Luigino, e moglie del segretario del fascio, della quale Levi aveva sottolineando alcuni aspetti in modo tale da suscitarne il profondo rancore, protrattosi fino alla morte di lei (1993). ${ }^{27}$

Viva rimane anche nella memoria degli alianesi l'immagine della Parroccola, figura di donna certamente di minor peso rispetto a quelle centrali della Santarcangelese o di Donna Caterina. Levi la descrive contrapponendo la sua estrema bruttezza fisica alla bontà d'animo, fornendone un ritratto incisivo e indimenticabile. ${ }^{28}$ Nonostante la descrizione che Levi fa del suo carattere, la Parroccola non gradì di essere citata dall'autore. Da tutto ciò si intende che, in un primo momento, quasi tutti i paesani vissero il Cristo come un'opera negativa. Non si serbavano buoni ricordi del passaggio dello scrittore torinese, né si manifestava alcun interesse nei suoi confronti, neanche da parte delle amministrazioni locali (Comune, Provincia, Regione).

Oggi sono poche le persone che tendono a riabilitare anche i personaggi negativi, come fa l'anziano farmacista, mentre la maggioranza approva i giudizi espressi da Levi su persone, eventi ed usanze. Per molti è giusto che Levi abbia descritto le condizioni di povertà, degrado e isolamento in cui versava il paese, la durezza della natura circostante e la fatica fatta per poterne trarre qualche frutto. Il suo scopo era quello di rendere evidente al mondo le condizioni di Aliano e di tutto il Sud Italia, affinché le pagine del libro potessero trasformarsi in uno stimolo ad agire. Aliano è ritratto come un paese fuori dalla storia, dove non «è arrivato il tempo, né l'anima individuale, né la speranza, né il legame tra le cause e gli effetti, la ragione e la Storia», ${ }^{29}$ è un paese immobile, ma tale descrizione serve proprio ad aiutarlo ad uscire dall'immobilità. ${ }^{30}$

Il fatto che, per anni — dall'uscita del Cristo fino alla morte dello scrittore e anche oltre- il ricordo di ciò che Levi aveva scritto rimanesse legato soprattutto a questa serie di opinioni negative, non ha impedito che lentamente la situazione si modificasse. Grazie al Cristo, per la prima volta Aliano e i suoi

26. Carlo LeVI, Cristo, cit., p. 47.

27. Angelo COLANGELO, op. cit., p. 35, «Pur riconoscendo a Carlo Levi qualche pregio sul piano umano $[\ldots]$, lo ha sempre contestato, per aver scritto nel suo libro famoso molte cose arbitrarie, che ne avevano ferito l'orgoglio".

28. Carlo Levi, Cristo, cit., p189. «La Parroccola, sua madre, così chiamata perché anch'essa aveva un grosso testone, che la faceva assomigliare al bastone pastorale del parroco, era una delle streghe contadine del paese: la piú modesta di tutte, la piú brutta e la piú bonaria. Quel suo faccione, con il largo naso piatto, le due enormi narici aperte, la boccaccia sgangherata, i capelli radi, la pelle ruvida e giallastra, era davvero mostruoso; e di corpo era piccola e tozza, infagottata negli stracci sotto il velo. Ma era una buona donna».

29. Carlo Levi, Cristo, cit., p. 3.

30. Carlo LeVI, Un volto che ci somiglia, cit, p. 103. «Immobile, ma "se abbiamo narrato di quel mondo immobile, era perché si muovesse", scrissi poi». 
abitanti fanno il loro ingresso nella Storia e diventano il fulcro di un'opera letteraria gravida di tante e così grandi conseguenze. ${ }^{31}$ Cristo si è fermato a Eboli è il documento che certifica agli occhi del mondo l'esistenza sia storica che geografica di Aliano, fornendogli una collocazione precisa, nonostante lo pseudonimo di Gagliano, e dal momento dell'uscita del libro (1945) mette il paese in comunicazione forte e durevole con la realtà esterna.

Il paese è, per così dire, fondato dal libro come successivamente farà, per la seconda volta, il Parco Letterario attraverso il lavoro di analisi del testo - anche se non sempre puntuale - estrapolandone gli elementi che, nella visione dei gestori culturali, caratterizzano più fortemente Aliano e la sua gente. Dopo un silenzio di secoli, nell'abisso temporale che separa la Magna Grecia da Carlo Levi, si può affermare che il Cristo rifonda la società alianese, dopo un rito di passaggio che condurrà alla comprensione e alla riappacificazione. Tale rito di passaggio, lungo e doloroso, è durato diversi decenni, fino a culminare nell'istituzione del Parco Letterario. La prova da superare era costituita dai sentimenti di ostilità e di rifiuto nei confronti del testo, dalla sofferenza di coloro che, a torto o a ragione, si ritenevano da esso ingiustamente colpiti. In un primo momento il fatto che il Cristo insistesse sul mondo magico e la peculiarità del tempo alianese, fece sentire agli abitanti del paese un senso di inadeguatezza, di un legame con l'arcaico che pesava su di un presente che si auspicava di sviluppo e di indiscutibile progresso. Gradualmente, con un'operazione lenta e collettiva, si è arrivati a comprendere il senso e le valenze del libro.

Attraverso la ricerca, lo studio e l'isolamento degli elementi utili, funzionali alla sua costituzione, il Parco Letterario, coadiuvato da tutta una serie di altri stimoli, —culturali, turistici, economici- ha monumentalizzato il testo, lo ha reso testimonianza di un epoca e di una condizione, lo ha assunto come punto di partenza per conferire un nuovo volto al paese.

Facendo un passo indietro, al primo impatto con il testo, gli alianesi avevano forse manifestato insofferenza perchè ritenevano che il Cristo fosse assunto all'esterno come documento assoluto, come l'unico modo di definirli assieme al loro ambiente. Ma, secondo un'interpretazione mutuata da Jacques Le Goff, il testo di Levi può essere inteso come il risultato di un montaggio scaturito dall'incontro dello scrittore, in un determinato momento storico, con la società con la quale egli è venuto in contatto. ${ }^{32}$

31. Raffaele GIURA Longo, Carlo Levi e «l'egemonia dei piccoli», p. 16: «Carlo Levi, Rocco Scotellaro ed Ernesto De Martino rappresentano, per noi lucani, l'espressione più alta, a livello culturale, di una felice stagione di crescita complessiva, civile e politica per la regione. La loro opera si intreccia con l'epoca delle lotte contadine degli anni '40- '50; è essa stessa frutto del vasto movimento delle campagne meridionali e, nel medesimo tempo, esprime la coscienza e le contraddizioni (cioè anche i problemi non risolti) di quel periodo. Attraverso di essa di può giungere alla giusta comprensione di aspetti importanti di un'intera fase della vita e della storia meridionale, così come anche grazie ad essa la vita e la storia del Mezzogiorno si saldano e si collegano alla vita e alla storia più generale e complessiva della società contemporanea».

32. Jacques Le Goff, Documento/monumento, in Enciclopedia Einaudi, vol. V, Torino: Einaudi, 1978, p. 46. «Il documento non è innocuo. È il risultato prima di tutto di un montag- 
Il Cristo è quindi prodotto dell'epoca in cui è stato scritto, ma anche risultato delle epoche ad essa successive, in cui ha continuato a vivere e a produrre degli effetti, e durante le quali non è mai stato in silenzio né manipolato dal silenzio. Probabilmente proprio il carattere di questa testimonianza forte, che veniva perpetuata indipendentemente dalla loro volontà, ha spinto gli alianesi a prendere posizione proprio perchè non desiderosi che la loro immagine fosse tramandata ai posteri alla maniera leviana.

Il Parco Letterario arriva dopo un lasso di tempo abbastanza lungo, tanto lungo da aver permesso alle polemiche e ai risentimenti di mitigarsi, dando la possibilità di una sorta di riscatto agli abitanti dell'antico luogo di confino. Con il Parco si ha la possibilità di isolare temi, valori, eventi, caratteri, personaggi che consapevolmente gli alianesi preposti alla guida dell'ente individuano come positivi, buoni da tramandare e da esportare.

Nel gioco di corrispondenze fra il modo di sentire Levi da parte degli alianesi e i valori che il Parco Letterario ha individuato e deciso di privilegiare, hanno preso corpo diverse narrazioni, spesso estrapolate dal Cristo e fuse con elementi recuperati dalla memoria. Il processo ha consentito la formazione di un bagaglio formulare, prodotto da una «memoria attiva ${ }^{33}$ intorno ai luoghi e ai personaggi, memoria che si è strutturata leggendo e rileggendo - letteralmente e non-, pensando, ascoltando ed elaborando.

Ad articolare le narrazioni - intese come mezzo per il recupero, l'organizzazione e la fissazione di ricordi ed eventi- hanno concorso sicuramente anche il trascorrere del tempo, le polemiche suscitate dal libro, i sentimenti dei singoli, l'introduzione di Cristo si è fermato a Eboli nei programmi scolastici e, negli ultimi anni, lo stesso Parco Letterario. Esso, dal momento della sua istituzione, ha svolto un ruolo fondamentale per la perpetuazione di tale memoria, come volano propulsore del bagaglio conoscitivo che ruota attorno a Carlo Levi e al Cristo. In questa maniera il Parco presiede alla conservazione e alla trasmissione del patrimonio comune.

La conservazione della «memoria buona» è scattata, dal momento in cui il testo leviano è stato assunto come mezzo di perpetuazione del patrimonio culturale del paese, diventando il racconto fondatore di Aliano. Carlo Levi costituisce per Aliano un patrimonio, un elemento che nel corso degli anni è stato incorporato ed assunto come caratterizzante della stessa comunità. La consapevolezza di poterne usufruire è emersa nell'ambito della collettività rinsal-

gio, conscio o inconscio, della storia, dell'epoca, della società che lo hanno prodotto, ma anche delle epoche successive durante le quali ha continuato a vivere, magari dimenticato, durante le quali ha continuato ad essere manipolato, magari dal silenzio. Il documento è una cosa che resta, che dura e la testimonianza, l'insegnamento [...] che reca devono essere in primo luogo analizzate demistificandone il significato apparente. Il documento è monumento. È il risultato dello sforzo compiuto dalle società storiche per imporre al futuro — volenti o nolenti- quella data immagine di se stesse. Al limite non esiste un documento — verità. Ogni documento è menzogna. Sta allo storico il non fare l'ingenuo».

33. Cfr., Mondher Kilani, La construction de la mémoire, Genève: Labor et Fides, 1992, p. 261. 
dando gradatamente il senso di identità degli abitanti ed offrendo inedite occasioni per un rinnovamento culturale ed economico del paese. Il fatto che tale presa di coscienza sia stata così lenta è dovuto anche alla prolungata convivenza con la memoria dello scrittore, negativa o positiva che fosse.

Per gli alianesi la permanenza di Levi ad Aliano, come confinato, era stata un avvenimento normale. Terminata quell'esperienza di confino Levi era tornato alla sua vita abituale, lontana da quei luoghi e da quelle persone, lasciando ricordi ed impressioni — soprattutto legate alla sua attività di medicoche dieci anni dopo sarebbero confluiti nella pubblicazione del Cristo e gli avrebbero consentito di fondersi con la quotidianità degli alianesi al punto di risultare quasi connaturato al paese. ${ }^{34}$

Oggi, superato il periodo in cui si negava la veridicità degli episodi narrati da Levi e in paese si contestava apertamente il libro, la quasi totalità degli intervistati dichiara di non trovare discordanze tra quella che è la propria conoscenza della storia del paese, dei suoi usi e tradizioni e quello che Levi descrive. Anzi, essi tengono a sottolineare la veridicità di tutto ciò che s'addensa nelle pagine del Cristo, e sembrano quasi voler cercare i punti in cui ritrovare descrizioni di oggetti, usi o avvenimenti che ricordano come esperienze vissute direttamente, che conoscono, quasi a volercisi riconoscere per ribadire il loro vincolo con Aliano, sostanziandolo nelle pagine dello scrittore torinese.

È possibile anche osservare il cambiamento nel rapporto fra gli abitanti del paese e il libro seguendo le poche visite che Carlo Levi fece ad Aliano successivamente al periodo di confino. Lo scrittore aveva espresso il desiderio di tornare ad Aliano già nelle prime righe di Cristo si è fermato a Eboli, ma non era riuscito a mantenere la promessa fatta ai «suoi contadini» per molto tempo. ${ }^{35}$ Il suo primo ritorno in paese si verificò in occasione delle elezioni della primavera del 1946, per il referendum istituzionale e l'Assemblea Costituente.

Nella campagna elettorale Carlo Levi si trovò di nuovo Basilicata, questa volta sostando in tanti dei paesi che avevano segnato i viaggi di andata e di ritorno verso il confino politico: Pisticci, Grassano, Tricarico, Garaguso, Accettura, Stigliano. L'ultima tappa fu Aliano. Poco prima di giungere in paese, Carlo Levi e il gruppo di politici che lo accompagnavano si trovarono immer-

34. Andrea Emiliani, Una politica dei beni culturali, Torino: Einaudi, 1974, p. 31. "La vastità materiale e concettuale del bene di cultura è, in Italia più che altrove, di proporzioni vitali, onnipresente e compresente ai modi stessi della nostra vita, nelle città come nelle campagne». Cfr. anche Carlo LEVI, «L'arte e gli italiani», in Le mille patrie. Uomini, fatti e paesi d'Italia, Roma: Donzelli, 2000, p. 24. «L'opera d'arte è motivo e origine di vita: si vive, spiritualmente, dentro le opere d'arte: ci si vive dentro anche materialmente». Poco più avanti, ibid. (p. 25), lo scrittore, parla dell'emozione che, in ogni luogo d'Italia, provoca la continua presenza di opere d'arte in chi non vi sia abituato e, di converso quanto gli abitanti del luogo siano invece abituati a convivere con antiche testimonianze del passato: «erano gli stessi luoghi che avevano visto le antiche guerre comunali, che erano stati nominati nelle terzine della Divina Commedia, questo esempio immortale della identità italiana fra i motivi dell'arte e quelli della vita. E ora come ribattezzati dai sacrifici e dal dolore, quei luoghi tornano giovani e presenti».

35. Carlo LeVI, Cristo, cit., p. 3. 
si nel fantastico paesaggio lunare dei calanchi. Nei pressi di Aliano, lo scrittore chiese di poter scendere dall'auto e di proseguire a piedi per poter riscoprire tutto, ogni casa, ogni pietra, per riprendere familiarità con ogni elemento e con le sensazioni che esso suscitava in lui, per risentire il rumore dell'acciottolato sconnesso e l'odore del paese. Grazie ad un veloce passaparola, non appena il gruppo di Levi si fermò ad ammirare il panorama, affacciandosi dalla Fossa del Bersagliere, accorse il vecchio podestà. Don Luigino salutò calorosamente l'ex confinato e lo rassicurò mettendogli una mano sulla spalla e affermando, rivolto soprattutto agli altri, di non avere nessun rancore nei confronti di Don Carlo. Aggiunse solamente che Levi avrebbe potuto citarlo diversamente nel Cristo, perché lui lo aveva sempre trattato bene, stimandolo e apprezzando la sua opera di pittore. Ormai però si poteva anche non parlarne più. Il discorso di Don Luigino esponeva la sua opinione davanti a tutti e servì a smorzare un po' i toni della polemica che era allora appena iniziata. ${ }^{36}$

Durante questo primo ritorno ad Aliano, Carlo Levi volle velocemente recarsi alla sua casa di confino. Presso la casa vi fu un rapido incontro con un contadino che lo ricordava ancora e che salutò tutti intrattenendosi a parlare e a illustrare i problemi del paese. Altre persone si fermarono a parlare con lo scrittore, venendogli incontro e stringendogli la mano. Tuttavia, da un angolo della piazza discretamente animata, dove stazionava un gruppetto di ragazzi, si levarono dei fischi. Complessivamente l'accoglienza però, non fu né pessima né molto calorosa. Le contestazioni nei suoi confronti non impedirono a Carlo Levi di portare a termine il suo giro per Aliano, «là dove aveva miticamente immaginato il cammino di liberazione del Mezzogiorno». ${ }^{37}$

Un altro momento importante nel mutamento del rapporto fra Aliano e Carlo Levi inizia circa trenta anni dopo l'uscita del Cristo, allorché, grazie alla più diffusa conoscenza del testo e alla scomparsa di molti dei personaggi che ne avevano operato una propaganda negativa, lo scrittore poté tornare ad Aliano in un clima migliore rispetto alla volta precedente. ${ }^{38}$

Con l'ultima visita si arriverà addirittura a un riavvicinamento, avvenuto grazie alla rielaborazione di tutta una serie di idee, sentimenti, riletture e sensazioni emersi dopo la morte dello scrittore, processo culminato nell' istituzione del Parco Letterario. Questa visita nel 1974 e fu possibile grazie anche a particolari condizioni politiche. Nel 1973, infatti, era stata eletta Sindaco, per il Partito Comunista (PCI), la giovane Maria Santomassimo, la prima donna sindaco nella storia della Basilicata.

Nel 1963 Carlo Levi era stato eletto senatore per il collegio di Civitavecchia, come candidato indipendente nelle liste del PCI; e nel 1968, rieletto,

36. Cfr., per la descrizione della visita, Leonardo SACCO, Il primo ritorno di Levi a "Gagliano», in Leonardo SACCO (a cura di), Contadini e Luigini, testi e disegni di Carlo Levi, RomaMatera: Basilicata editrice, 1975, p. 22-26.

37. Gigliola De Donato, Sergio D’Amaro, Un torinese del Sud, cit., p. 175.

38. Oltre a la visita qui descritta, avvenuta nel maggio 1946, Levi tornò ad Aliano in altre due occasioni, nel marzo 1955 nel dicembre 1974, pochi mesi prima di morire. Cfr. Gigliola De Donato p. 354, 355 e 358. 
questa volta nel collegio senatoriale di Velletri, nella lista Pci-Psiup (Partito Socialista Italiano di Unità Proletaria), aveva aderito al gruppo della sinistra indipendente. Anche in virtù della comune militanza e ormai sicura della buona accoglienza che la popolazione avrebbe riservato allo scrittore, la giovane Sindaco procedette ad un invito ufficiale. Carlo Levi si recò così ad Aliano fra il 7 ed il 10 dicembre del 1974, poco meno di un mese prima della sua scomparsa.

La morte dello scrittore fu seguita dalla decisione, presa congiuntamente dalla famiglia e dal comune, di seppellirlo ad Aliano. Tutto ciò ha inciso fortemente sul modo di tramandare la memoria di Levi in paese. Con la sepoltura nel piccolo cimitero, e quindi con la presenza del suo corpo, lo scrittore è legato permanentemente alla memoria del paese, entra a farne parte integrante. I ricordi relativi alla sua vita — soprattutto quelli degli otto mesi di confino trascorsi ad Aliano - si intrecciano con quelli del paese, permettendo la reciproca fissazione in due memorie differenti.

Il fatto che le spoglie di Carlo Levi riposino nel piccolo cimitero sabbioso consente di recuperare ulteriormente la "memoria buona» che è presente in Cristo si è fermato a Eboli. Tale memoria permette di mantenere in vita il paese, garantendo la permanenza dell'identità individuale e di gruppo, in nome dei ricordi positivi fissati dalle pagine del testo contro il rischio di un decisivo smarrimento che avrebbe comportato e comporterebbe la dissoluzione di Aliano in una massa indistinta di luoghi, geograficamente e storicamente indefinita. Carlo Levi è legato così, in maniera molto forte, alla memoria collettiva del paese, mentre Aliano, grazie alla presenza dello scrittore, entra a far parte di una memoria, più ampia, nazionale e letteraria.

La morte di Levi rende nuovamente presente in paese il suo corpo, ormai senza vita; l'atto stesso del suo seppellimento diviene paragonabile agli antichi riti di fondazione di un edificio, strutturato su basi solide e capace di sfidare il tempo. Con la sua presenza permanente ad Aliano Carlo Levi ricostruisce il tempio della memoria alianese e, attraverso il Cristo, la rende perpetua. 